\title{
Quantitative Immuno-SPECT Monitoring of Pretargeted Radioimmunotherapy with a Bispecific Antibody in an Intraperitoneal Nude Mouse Model of Human Colon Cancer
}

\author{
Rafke Schoffelen ${ }^{1}$, Winette T.A. van der Graaf $^{2}$, Robert M. Sharkey ${ }^{3}$, Gerben M. Franssen ${ }^{1}$, William J. McBride ${ }^{4}$, \\ Chien-Hsing Chang ${ }^{5}$, Desirée L. Bos ${ }^{1}$, David M. Goldenberg ${ }^{3-5}$, Wim J.G. Oyen ${ }^{1}$, and Otto C. Boerman ${ }^{1}$ \\ ${ }^{I}$ Department of Nuclear Medicine, Radboud University Nijmegen Medical Centre, Nijmegen, The Netherlands; ${ }^{2}$ Department \\ of Medical Oncology, Radboud University Nijmegen Medical Centre, Nijmegen, The Netherlands; ${ }^{3}$ Garden State Cancer Center, \\ Center for Molecular Medicine and Immunology, Morris Plains, New Jersey; ${ }^{4}$ Immunomedics, Inc., Morris Plains, New Jersey; \\ and ${ }^{5}$ IBC Pharmaceuticals, Morris Plains, New Jersey
}

The prospects for using pretargeted immuno-SPECT to monitor the response to pretargeted radioimmunotherapy were examined. In this study, a bispecific anticarcinoembryonic antigen (CEACAM5; CD66e) $\times$ antihapten monoclonal antibody, TF2, was used in combination with a small (1.5 kD) peptide, IMP288, labeled with ${ }^{111}$ In and ${ }^{177} \mathrm{Lu}$. Methods: First, tumor uptake of ${ }^{111} \mathrm{In}$-IMP288 and ${ }^{177} \mathrm{Lu}$-IMP288, as determined by immuno-SPECT, was validated by ex vivo counting. Two groups of female BALB/C nude mice had LS174T tumors implanted in the peritoneal cavity. They received intravenous injections of TF2, followed by $10 \mathrm{MBq}$ of ${ }^{111} \mathrm{In}-\mathrm{IMP} 288$ or 90 $\mathrm{MBq}$ of ${ }^{177} \mathrm{Lu}-\mathrm{IMP} 288$. A control group of non-tumor-bearing mice received TF2 and ${ }^{111} \mathrm{In}-\mathrm{IMP} 288$. One hour after the radiolabeled IMP288 was given, small-animal SPECT/CT images were acquired, and subsequently animals were dissected. Furthermore, a survival study was performed in 3 groups of 10 mice with intraperitoneal tumors: mice received TF2 and ${ }^{177} \mathrm{Lu}-$ IMP288 (60 MBq), nonpretargeted ${ }^{177} \mathrm{Lu}-\mathrm{IMP} 288$ (60 MBq), or phosphate-buffered saline. Immuno-SPECT scans were acquired directly after therapy and at 14 and $45 \mathrm{~d}$ after therapy. Tumor growth was analyzed in the successive scans in each animal. Results: ${ }^{111} \mathrm{In}$ - and ${ }^{177} \mathrm{Lu}$-labeled IMP288 had similar in vivo distribution. The activity measured in the pretargeted immuno-SPECT images correlated well with the uptake measured in the dissected tumors (Pearson $r=0.99, P<0.05$ ). In the therapy study, the SPECT images showed rapid and selective tumor targeting with high tumor-to-background contrast $(30 \pm 12)$ as early as $1 \mathrm{~h}$ after injection. The successive images of the treated mice showed delayed tumor growth in the pretargeted radioimmunotherapy group, corresponding with their prolonged survival. Conclusion: Pretargeted immuno-SPECT with TF2 and ${ }^{111}$ In- or ${ }^{177}$ Lu-IMP28 8 can be used to predict and confirm tumor targeting and monitor the therapeutic effect of pretargeted radioimmunotherapy.

Received Mar. 21, 2012; revision accepted Jul. 2, 2012.

For correspondence or reprints contact: Rafke Schoffelen, Radboud University Nijmegen Medical Centre, Department of Nuclear Medicine, P.O.

Box 9101, 6500 HB Nijmegen, The Netherlands.

E-mail: r.schoffelen@nucmed.umcn.nl

Published online Oct. 18, 2012.

COPYRIGHT @ 2012 by the Society of Nuclear Medicine and Molecular Imaging, Inc.
Key Words: SPECT; pretargeting; bispecific antibody; mice; LS174T; radioimmunotherapy

J Nucl Med 2012; 53:1926-1932

DOI: 10.2967/jnumed.112.106278

$\mathbf{R}$ adiolabeled antibodies can be used for the diagnosis, detection, and therapy of cancer. The diagnostic images acquired after injection of the radiolabeled antibody preparation can potentially predict the efficacy of radioimmunotherapy, an approach designated as theranostics. Theranostics is a useful concept for developing personalized targeted radionuclide therapies and has been applied for a long time in nuclear medicine. For example, diagnostic information obtained from pretherapeutic PET or SPECT can ensure that the treatment is targeted specifically to tumors. This kind of information could aid in patient selection, and dosimetric analysis potentially could predict the benefit-to-risk ratio of a planned radionuclide therapy. This analysis also might aid in identifying the most appropriate prescribed therapeutic dose optimized for a patient's radionuclide therapy (e.g., ${ }^{90} \mathrm{Y},{ }^{177} \mathrm{Lu}$, or ${ }^{213} \mathrm{Bi}$ ). Finally, imaging data could potentially assess therapeutic response, including the detection of unsuspected sites of disease. The feasibility of this approach has been shown in neuroendocrine tumors using ${ }^{111} \mathrm{In}$ - or ${ }^{68} \mathrm{Ga}-$ labeled somastatin analogs for diagnosis and the same peptides labeled with ${ }^{177} \mathrm{Lu}$ or ${ }^{90} \mathrm{Y}$ for radionuclide therapy $(1,2)$.

In this study, the potential of combined pretargeted radioimmunodetection and radioimmunotherapy was evaluated. Pretargeting is a strategy that was developed to improve the imaging and therapeutic characteristics of directly radiolabeled monoclonal antibodies. Radiolabeled antibodies require several days to localize tumors effectively, because of the slow pharmacokinetics and accretion of intact antibodies in tumors. This slow uptake and clearance rate delay tumor detection by imaging and increase bone marrow toxicity for therapy. Pretargeting techniques achieve rapid accretion of 
the radionuclide in the tumor in combination with rapid blood clearance by first administering a nonradiolabeled bispecific monoclonal antibody (bsmAb). After the bsmAb localizes in the tumor and clears from the circulation, a radiolabeled hapten peptide is given. This small molecule leaves the bloodstream quickly, being trapped in the tumor by the antihapten arm of the bsmAb. Its remainder clears rapidly from the blood and is excreted via the kidneys. Coupling 2 haptens to the peptide improves peptide uptake, a phenomenon known as affinity enhancement (3). Chelate-metal complexes, such as diethylenetriaminepentaacetic acid-indium, have been used as haptens (4). More recently, peptides substituted with the hapten histamine-succinyl-glycine (HSG), in combination with anti-HSG antibody binding, have provided a more flexible system, because these HSG-substituted peptides can be conjugated with various chelators (e.g., DOTA, NODA/NOTA, or $\mathrm{N}_{3} \mathrm{~S}$-chelates), allowing stable complexes with many radionuclides, such as ${ }^{111} \mathrm{In}$ and ${ }^{99 \mathrm{~m} T c}$ for SPECT $(5-8),{ }^{68} \mathrm{Ga}$ and ${ }^{18} \mathrm{~F}$ for PET (9-13), or ${ }^{90} \mathrm{Y}$ and ${ }^{177} \mathrm{Lu}$ for pretargeted radioimmunotherapy (PRIT) $(5,8)$. Including tyrosine in the peptide core also enables radioiodination (e.g., ${ }^{124} \mathrm{I}$ or $\left.{ }^{131} \mathrm{I}\right)(7,14)$.

For the studies described herein, a fully humanized anticarcinoembryonic antigen $\times$ anti-HSG bsmAb (TF2) (15) and an HSG-substituted hapten peptide (IMP288) were applied in a nude mouse model for peritoneal dissemination of human cancer (13). Previous preclinical studies illustrated the enhanced sensitivity and specificity of this pretargeting system, compared with ${ }^{18}$ F-FDG PET $(7,12,13)$.

The aim of the present study was to investigate whether pretargeted immuno-SPECT could monitor the effectiveness of PRIT.

\section{MATERIALS AND METHODS}

\section{Pretargeting Reagents TF2 and IMP288}

The bsmAb, TF2, and the peptide IMP288 were provided by Immunomedics and IBC Pharmaceuticals. The preparation of TF2 and its binding properties have been described previously $(15,16)$. Gel filtration chromatography showed that TF2 bound more than $90 \%$ of radiolabeled peptide.

IMP288 was synthesized and purified as described by McBride et al. (14). It is a DOTA-conjugated D-Tyr-D-Lys-D-Glu-D-Lys tetrapeptide in which both lysine residues are substituted with an HSG moiety via their $\varepsilon$-amino group: DOTA-D-Tyr-D-Lys(HSG)D-Glu-D-Lys(HSG)- $\mathrm{NH}_{2}$.

IMP288 was labeled with ${ }^{111}$ In (Covidien) at a specific activity of $36 \mathrm{MBq} / \mathrm{nmol}$ and with ${ }^{177} \mathrm{Lu}$ (IDB Holland BV) at a specific activity of $321 \mathrm{MBq} / \mathrm{nmol}$, under strict metal-free conditions. ${ }^{111} \mathrm{In}$ $(100 \mathrm{MBq})$ was added to $4 \mu \mathrm{g}(2.8 \mathrm{nmol})$ of IMP288, or $900 \mathrm{MBq}$ of ${ }^{177} \mathrm{Lu}$ were added to $4 \mu \mathrm{g}(2.8 \mathrm{nmol})$ of IMP288. Then the mixture was dissolved in $0.1 \mathrm{M} 2$-( $\mathrm{N}$-morpholino)ethanesulfonic acid buffer, $\mathrm{pH} 5.5$, and incubated for $20 \mathrm{~min}$ at $95^{\circ} \mathrm{C}$ in a heating block. Subsequently, $10 \mu \mathrm{L}$ of $50 \mathrm{mM}$ ethylenediaminetetraacetic acid were added in order to complex any unbound ${ }^{111} \mathrm{In}$ or ${ }^{177} \mathrm{Lu}$. Radiochemical purity of the radiolabeled IMP288 preparations was determined by reversed-phase high-performance liquid chromatography as described previously (5). In all experiments, the radiochemical purity of radiolabeled IMP288 exceeded 95\%.
Mice received TF2 and IMP288 intravenously in $0.2-0.3 \mathrm{~mL}$ of phosphate-buffered saline (PBS) and $0.5 \%$ bovine serum albumin.

\section{Animal Experiments}

All studies were approved by the institutional Animal Welfare Committee of the Radboud University Medical Centre Nijmegen and conducted in accordance with their guidelines (revised Dutch Act on Animal Experimentation, 1997). The experiments were performed in female nude BALB/c mice (6-8 wk old) weighing 20$25 \mathrm{~g}$. Mice were acclimated to laboratory conditions for at least $1 \mathrm{wk}$ before experimental use. They were housed under nonsterile standard conditions in filter-topped cages with free access to animal chow and water. Tumor growth was induced by an intraperitoneal injection of $0.5 \mathrm{~mL}$ of a suspension of $10^{6} \mathrm{LS} 174 \mathrm{~T}$ cells, a carcinoembryonic antigen-expressing human colon carcinoma cell line (CCL188; passage 7; American Type Culture Collection), resulting in tumor nodules $(1-3 \mathrm{~mm})$ in the peritoneal cavity after $2-4 \mathrm{wk}(17)$.

\section{Biodistribution and Imaging Studies}

The aim was to investigate whether ${ }^{111}$ In-IMP288 could be used as a surrogate for ${ }^{177} \mathrm{Lu}$-IMP288 with pretargeted immuno-SPECT. Therefore, the biodistribution of ${ }^{111}$ In-IMP288 and ${ }^{177} \mathrm{Lu}-\mathrm{IMP} 288$ was determined by small-animal SPECT and by the counting of dissected tumors.

Two groups of 5 nude mice with intraperitoneal tumors and 1 group of 3 mice without tumors (negative control) were injected intravenously with $5.0 \mathrm{nmol}$ of TF2 $(0.2 \mathrm{~mL})$. Sixteen hours later, $0.28 \mathrm{nmol}$ of IMP288 $(0.2 \mathrm{~mL})$ was administered intravenously. One group received ${ }^{111} \mathrm{In}$-labeled IMP288 (10 MBq), and the other group received ${ }^{177} \mathrm{Lu}$-labeled IMP288 $(90 \mathrm{MBq})$. One hour after injection of the radiolabeled peptide, mice were euthanized by $\mathrm{CO}_{2}-\mathrm{O}_{2}$ asphyxiation. The control group of mice without any tumor received the same dose of TF2 and ${ }^{111}$ In-IMP288. Before imaging, the total-body activity of each mouse was measured in a dose calibrator. A blood sample $(50-100 \mu \mathrm{L})$ was drawn by cardiac puncture. Mice were imaged using an USPECT-II/CT scanner (MILabs) with a 1.0-mm-diameter pinhole rat collimator tube. The animals were placed supine. SPECT scans were acquired for $60 \mathrm{~min}$, followed by CT scans for anatomic reference ( $65 \mathrm{kV} ; 612 \mu \mathrm{A}$; exposure time, $240 \mathrm{~ms}$ ).

After imaging, mice were dissected, and the abdomen was systematically and meticulously examined for the presence of tumors. The location of each lesion was documented and individually weighed, lesion size was measured, and the radioactivity was detected in a $\gamma$ counter. The other organs of interest (liver, spleen, pancreas, intestines, kidney, lung, and muscle) were weighed and counted in a $\gamma$-counter with standards prepared from the injected products, using appropriate energy windows for the radionuclide of interest. The percentage of the injected dose per gram of tissue $(\% \mathrm{ID} / \mathrm{g})$ was calculated.

\section{Image Analysis}

Scans were reconstructed with software from MILabs, using an ordered-subset expectation maximization algorithm with a voxel size of $0.375 \mathrm{~mm}$. Images were analyzed with the Inveon Research Workplace software (version 2.2; Siemens Preclinical Solutions). Images were scored for the presence, number, and localization of intraperitoneal tumors.

To estimate the absolute tumor uptake (\%ID) from the images, calibration factors for ${ }^{111} \mathrm{In}$ and ${ }^{177} \mathrm{Lu}$ were determined; whole-body activity as measured in the scans was correlated to the activity measured in the animals measured in the dose calibrator. Volumes of 
interest (VOIs) were drawn around the tumor lesions. A VOI in the lower left abdomen (large intestines) was used as background activity. The activity concentrations in the tumors derived from the images were compared with the tumor uptake as measured in the $\gamma$-counter. A tissue density of $1.0 \mathrm{~g} / \mathrm{mL}$ was assumed.

\section{Pretargeted Radioimmunotherapy Studies}

The design of the PRIT study is summarized in Figure 1. Three groups of 10 mice were inoculated intraperitoneally with LS174T tumor cells. Two weeks after tumor cell inoculation, mice were given $5.0 \mathrm{nmol}$ of TF2, followed $16 \mathrm{~h}$ later with $60 \mathrm{MBq}$ of ${ }^{177} \mathrm{Lu}-\mathrm{IMP} 288$ (previously determined to be the maximum-tolerated dose). The 2 control groups received PBS or $60 \mathrm{MBq}$ of ${ }^{177} \mathrm{Lu}$-IMP288 without the pretargeting of TF2 on the same day that the treatment group received radiolabeled IMP288.

One hour after the PRIT group received the ${ }^{177} \mathrm{Lu}$-IMP-288, a baseline immuno-SPECT scan was acquired. The control groups were scanned after a diagnostic injection of ${ }^{111}$ In-labeled IMP288 pretargeted with TF2, $1 \mathrm{~d}$ after therapy (Fig. 1). Follow-up scans were acquired in all remaining animals of all groups at 14 and $45 \mathrm{~d}$ after therapy, after a diagnostic injection of ${ }^{111}$ In-labeled IMP288 pretargeted with TF2. Animals were scanned under general anesthesia (isoflurane and $\mathrm{O}_{2}$ ). The same imaging acquisition protocol, reconstruction, and calibration were used as in the biodistribution and imaging studies. The scans were analyzed by the drawing of VOIs around tumor lesions. The fraction of the administered dose was calculated. By comparing the measurements of the same lesions in the follow-up scan and in the baseline scan, growth of each tumor lesion was monitored.

Animals were observed daily by independent, experienced biotechnicians. Humane endpoints were a mouse's failure to eat or drink and the loss of $15 \%$ or more of body weight in 1 or $2 \mathrm{~d}$, the loss of $20 \%$ or more of the mouse's starting weight, or tumor progression-abdominal distention or visual hemorrhagic ascites. When the humane endpoint was reached, mice were euthanized by $\mathrm{CO}_{2}-\mathrm{O}_{2}$ asphyxiation. All remaining animals were euthanized at $120 \mathrm{~d}$ after therapy.

\section{Statistical Analysis}

Biodistribution, SPECT, and survival data were statistically analyzed using GraphPad Prism (version 5.00; GraphPad Software)

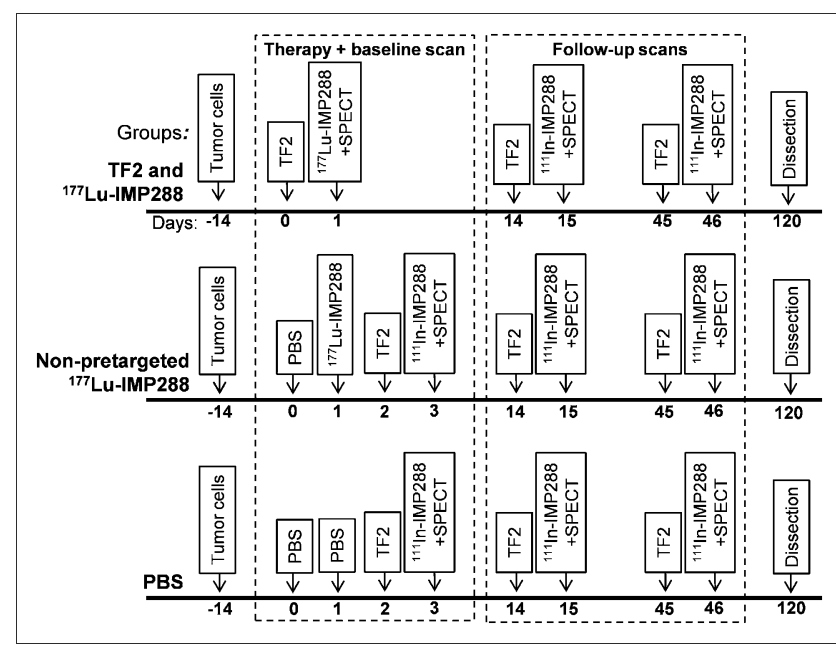

FIGURE 1. Treatment schedule for 3 different therapy groups and timing of baseline and follow-up SPECT studies. for Windows (Microsoft). The mean and SD were used to describe continuous data, unless stated otherwise. Comparisons were analyzed using a nonparametric, 2-tailed Mann-Whitney test. Correlations were determined with the Spearman correlation test. Survival was described with median survival times, and survival curves were compared using the log-rank test. The level of significance was set at a $P$ value less than 0.05 .

\section{RESULTS}

\section{Biodistribution and Imaging Study}

Mice did not show clinical signs of discomfort or a change in body weight for up to $3 \mathrm{wk}$ after inoculation of the LS174T tumor cells. Nevertheless, on macroscopic inspection of their abdomen, multiple solid tumors were found, predominantly located at the rectovesical pouch; mesentery; and subhepatic, -splenic, and -phrenic spaces. The median number of tumor lesions per animal was 5 (range, 0-9). Three-dimensional caliper measurements revealed that the median diameter of the lesions was $5 \mathrm{~mm}$ (range, 1-16 mm), corresponding to a median weight of $22 \mathrm{mg}$ (range, $0.6-840 \mathrm{mg}$ ). Two of 10 mice did not develop any macroscopically visible tumors.

The biodistribution of ${ }^{111}$ In-IMP288 and ${ }^{177} \mathrm{Lu}-\mathrm{IMP} 288$ at $1 \mathrm{~h}$ after injection in the mice that were pretargeted with TF2 was similar (Fig. 2). Uptake of ${ }^{111} \mathrm{In}-\mathrm{IMP} 288$ and ${ }^{177} \mathrm{Lu}-$ IMP288 in the tumor lesions was similar $(6.9 \pm 2.7 \% \mathrm{ID} / \mathrm{g}$ vs. $7.1 \pm 2.7 \% \mathrm{ID} / \mathrm{g}$, respectively; Mann-Whitney, $P>$

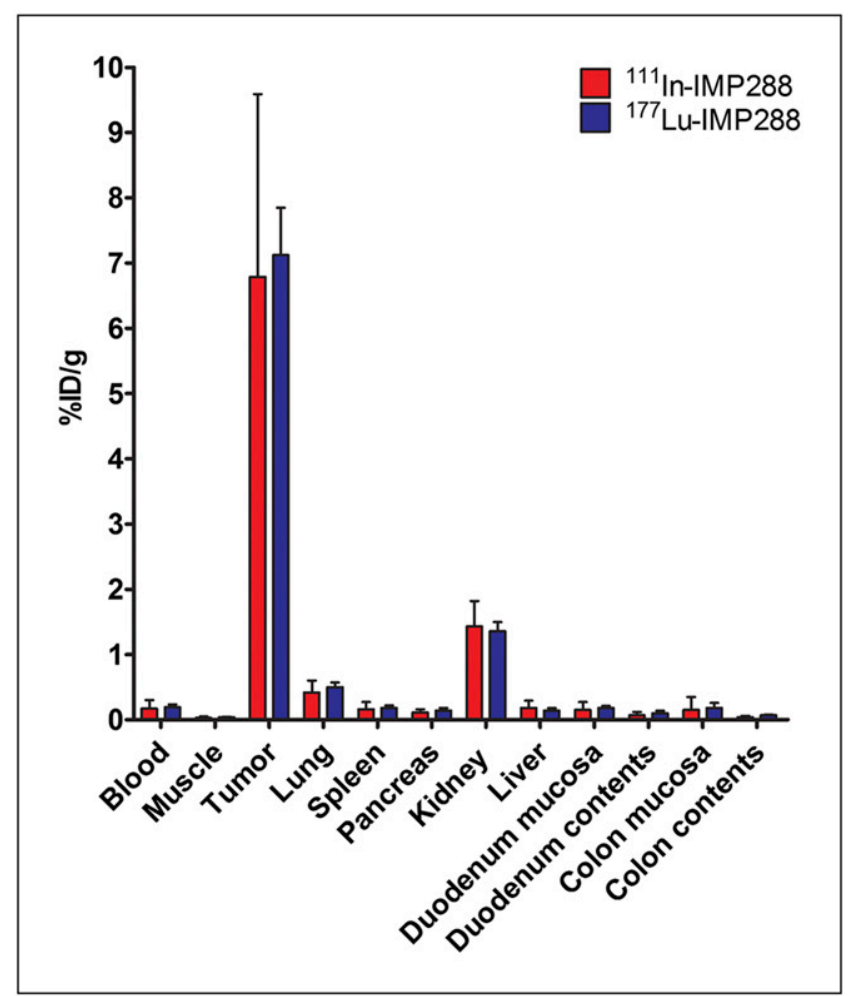

FIGURE 2. Biodistribution of $0.25 \mathrm{nmol}$ of ${ }^{111} \mathrm{In}$ - or ${ }^{177} \mathrm{Lu}$-IMP288, after pretargeting with $6.0 \mathrm{nmol}$ of ${ }^{125}$-TF2 (interval, $16 \mathrm{~h}$ ), $1 \mathrm{~h}$ after intravenous injection, in BALB/c nude mice with intraperitoneal, carcinoembryonic antigen-expressing LS174T tumors. Values are given as mean $\pm \mathrm{SD}(n=5)$. 
0.05). Likewise, blood concentrations at $1 \mathrm{~h}$ after injection were the same $(0.17 \pm 0.13 \% \mathrm{ID} / \mathrm{g}$ vs. $0.16 \pm 0.08 \% \mathrm{ID} / \mathrm{g}$, respectively; Mann-Whitney, $P>0.05$ ). At $1 \mathrm{~h}$ after injection, the ratios of uptake in the tumor to that in the other organs were $31.0 \pm 30.8$ (blood), $66.2 \pm 23.4$ (intestine), $3.7 \pm$ 2.4 (kidney), and $27.4 \pm 20.5$ (liver) for ${ }^{111}$ In-IMP288 and $34.6 \pm 11.4$ (blood), $58.0 \pm 18.0$ (intestine), $4.2 \pm 0.3$ (kidney), and $40.4 \pm 49.1$ (liver) for ${ }^{177} \mathrm{Lu}-\mathrm{IMP} 288$. Normaltissue biodistribution of ${ }^{111}$ In-IMP288 in the non-tumorbearing mice was not significantly different from that of the tumor-bearing animals (data not shown), indicating that the presence of the intraperitoneally growing tumors did not affect clearance of the peptide from the normal tissues.

\section{Image Analysis}

Clear and specific targeting of the tumor lesions was seen in SPECT images that were acquired at $1 \mathrm{~h}$ after injection of ${ }^{111} \mathrm{In}$ - and ${ }^{177} \mathrm{Lu}$-IMP288. A typical immuno-SPECT image of a tumor-bearing mouse is shown in Figure 3A. The corresponding photographs show all the tumors that were dissected from the abdomen of this animal. Each lesion was clearly visualized in the SPECT images. An example of a SPECT image of a non-tumor-bearing mouse is shown in Figure 3B, demonstrating renal uptake and only low accretion in the other normal organs. Normal-tissue uptake was low in most of the tumor- and non-tumor-bearing mice. In some mice, low uptake in the spleen (3/15), uterus and ovaries (2/15), and intestines (2/15) was seen, which could easily be discriminated from tumor lesions by its activity concentration, shape, and location.

Tumor-to-background ratios were high $(30 \pm 12)$ using the lower left abdomen as a background region. The ratios did not differ between the groups that received the ${ }^{111} \mathrm{In}$ - or ${ }^{177} \mathrm{Lu}$-labeled peptide (33 \pm 15 vs. $27 \pm 9$; Mann-Whitney,

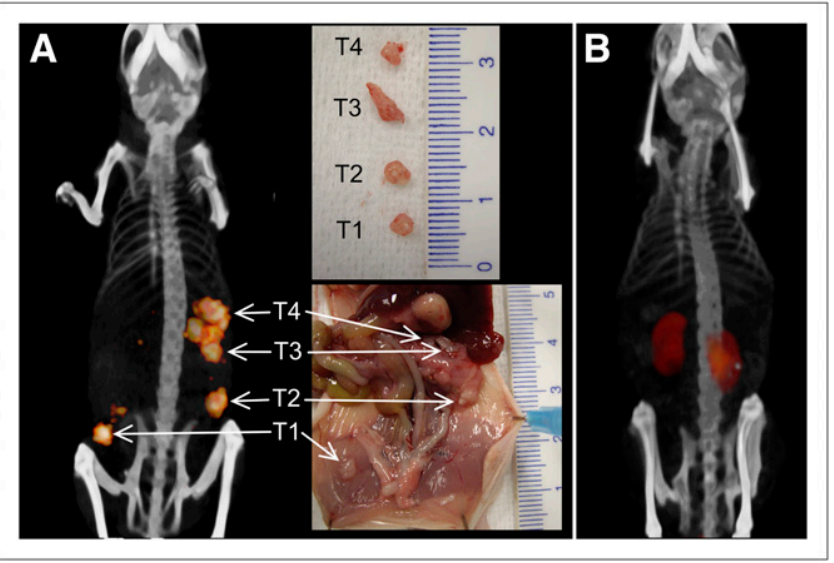

FIGURE 3. Three-dimensional-volume rendering of pretargeted immuno-SPECT/CT images of tumor-bearing mouse (A) and nontumor-bearing mouse (B). Animals were imaged at $1 \mathrm{~h}$ after ${ }^{111} \mathrm{In}$ IMP288 injection. Corresponding digital photographs were taken during dissection to localize and measure individual tumors. All dissected tumors (T1-T4) are clearly distinguishable on pretargeted immuno-SPECT/CT images, with low normal-tissue uptake.
$P>0.05$ ). All lesions $2 \mathrm{~mm}$ or greater in diameter ( $\geq 4 \mathrm{mg}$ ) were detected as separate lesions with immuno-SPECT.

Tumor uptake, as determined by immuno-SPECT and ex vivo counting, showed a good correlation (Pearson $r=0.99$, $P<0.05$; Fig. 4). Furthermore, the absolute values (\%ID) of both methods showed good agreement, with a mean difference of only $13 \% \pm 38 \%$ (Bland-Altman analysis). In all tumors $22 \mathrm{mg}$ or more (median tumor weight), the difference between the 2 measurements was less than $25 \%$. In smaller tumors, the uptake measured with immuno-SPECT was less reliable, where uptake measured by SPECT in $40 \%$ of the lesions was more than $25 \%$ different.

\section{PRIT Studies}

Tumor Growth. In 2 animals in the PBS group and in 3 animals in the PRIT group, the baseline or follow-up scans did not disclose any tumors. These animals did not show any tumors at dissection $120 \mathrm{~d}$ after tumor inoculation. In most of the animals, small-animal SPECT scans directly after therapy revealed multiple tumor lesions per animal: the median number of visualized tumors per animal was 4 (range, 1-10). One mouse in the PBS group had to be euthanized before the first follow-up scan (14 d after injection), because of excessive tumor growth.

Tumor growth was monitored with immuno-SPECT after therapy by measuring the fraction of the injected dose of ${ }^{111} \mathrm{In}$ - and ${ }^{177} \mathrm{Lu}-\mathrm{IMP} 288$ for each lesion, drawing VOIs around the tumors in the baseline and in the follow-up scans. Figure 5 shows a typical set of scans of an animal from the control group that received nonpretargeted ${ }^{177} \mathrm{Lu}-\mathrm{IMP} 288$. Tumor activity concentrations were similar in both scans,

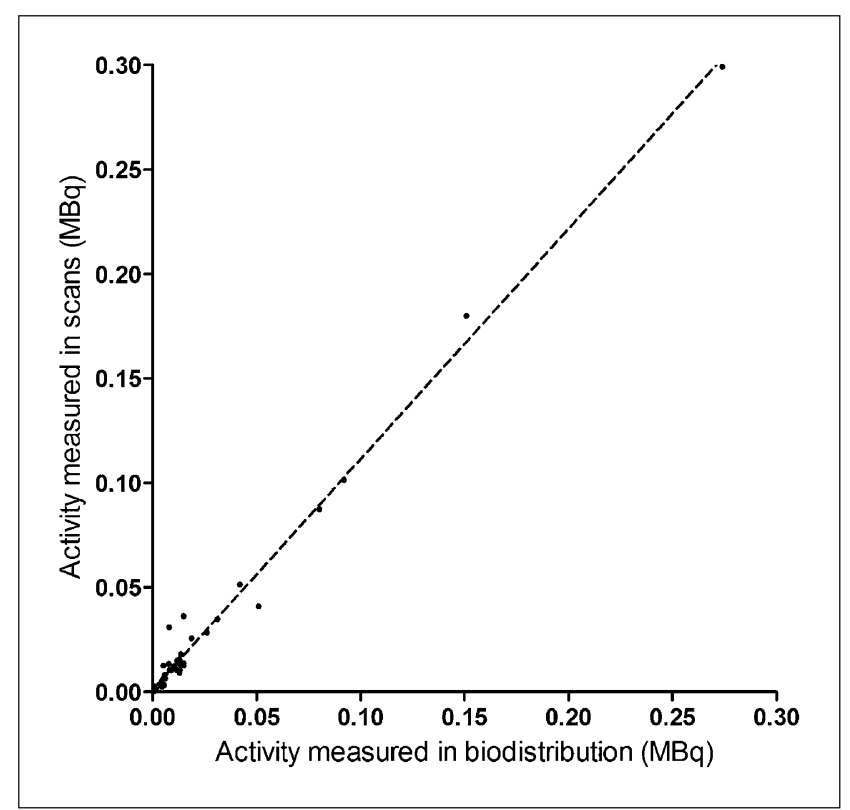

FIGURE 4. Correlation between tumor uptake measured in pretargeted immuno-SPECT images ( $y$-axis) and by ex vivo biodistribution ( $x$-axis) (Spearman $\rho=0.99, P<0.05)$. Values are given for individual tumor lesions. Dotted line is linear fitted trend line. 
but the fraction of the injected dose per lesion was 10-fold higher in the follow-up scan on day 14 (Figs. 5A and 5B). The animal had to be euthanized because of abdominal distension and ascites $9 \mathrm{~d}$ after the follow-up scan. Dissection of this animal revealed $2.0 \mathrm{~g}$ of tumor tissue (Fig. 5C). Thus, the excessive tumor growth that was prospectively monitored by imaging was confirmed ex vivo.

PRIT resulted in a significant delay in tumor growth. Tumor growth inhibition was observed in the follow-up scans at $14 \mathrm{~d}$ after therapy. Tumor uptake calculated as a fraction of the administered dose increased 13- to 16-fold in the nonpretargeted group and the PBS group, respectively (nonpretargeted group: mean, 1.289\%, and SEM, 154\%; PBS group: mean, $1.584 \%$, and SEM, 167\%). In contrast, in the PRIT group a 5-fold increase (535\%; SEM, 118\%) in tumor growth was observed at $14 \mathrm{~d}$ after therapy (Mann-Whitney, $P<0.05$ ) (Fig. 6). At the time of the second follow-up scan (45 d after therapy), only 1-3 mice per group were still alive.

Survival. Delayed tumor growth due to PRIT resulted in a median survival of $50 \mathrm{~d}$ (95\% confidence interval, 41-59 d), whereas the mice in the nonpretargeted group and the PBS group had a median survival of 22 and $19 \mathrm{~d}$, respectively (95\% confidence interval, 15-29 and 9-29 d, respectively) (Fig. 7). Kaplan-Meier survival analysis showed that the overall survival curve of the groups treated with PRIT was significantly better than the overall survival of the group not treated with pretargeted peptide $(P<0.05)$. All animals in the PBS and nonpretargeted group had to be euthanized because of tumor progression; animals presented either with abdominal distension or with ascites. In the treated animals that had to be euthanized in the same period $(<4 \mathrm{wk}$ after therapy), the main reason for euthanasia was loss of body

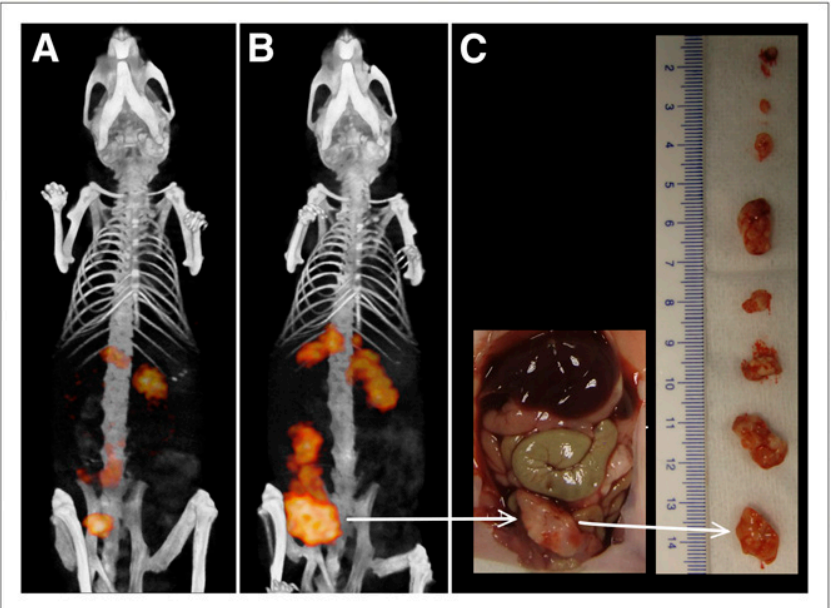

FIGURE 5. Three-dimensional-volume rendering of pretargeted immuno-SPECT/CT images of mouse that was treated with nonpretargeted ${ }^{177} \mathrm{Lu}$-IMP288 at time of therapy (A) and follow-up scan at $14 \mathrm{~d}$ after therapy $(\mathrm{B})$, which showed 10 -fold increase in tumor size in this animal. Corresponding digital photographs (C) were taken when animal had to be euthanized $9 \mathrm{~d}$ after follow-up scan and show multiple tumors (total weight, $2 \mathrm{~g}$ ) that were dissected from abdomen. Arrows indicate 1 tumor.

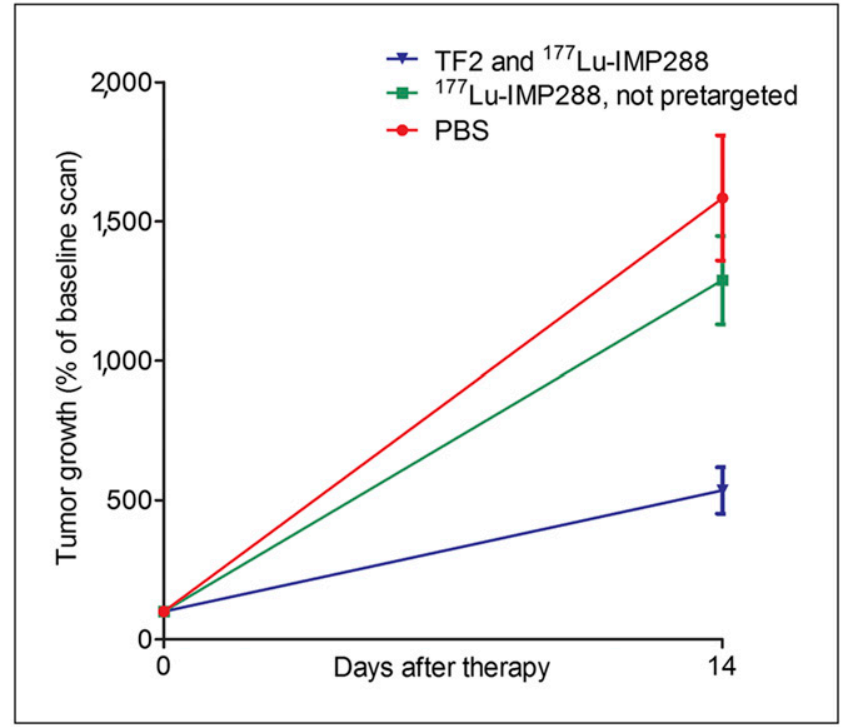

FIGURE 6. Difference in tumor growth between therapy groups (TF2 and ${ }^{177}$ Lu-IMP288, nonpretargeted ${ }^{177}$ Lu-IMP288, or PBS), measured in pretargeted immuno-SPECT images at time of therapy and follow-up scan made $14 \mathrm{~d}$ after therapy. Difference was calculated as percentage increase in fraction of administered dose. Values are given as mean \pm SEM of all individual tumors per therapy group.

weight, whereas at dissection they had a low total tumor weight (9-fold lower than in the control animals).

Two animals in the PBS group and 3 in the PRIT group survived for more than $120 \mathrm{~d}$ after therapy. None of these animals had tumors visible in the scans or at dissection (134 d after tumor inoculation). As a result, Kaplan-Meier survival analysis of those 2 curves did not show significant differences.

\section{DISCUSSION}

The present study shows that pretargeted immuno-SPECT is an excellent imaging method for monitoring the therapeutic effect of PRIT or, for that matter, monitoring any form of therapy. The biodistribution showed that the ${ }^{111} \mathrm{In}$ labeled peptide is a reliable surrogate for the ${ }^{177} \mathrm{Lu}$-labeled peptide. Pretargeted immuno-SPECT with TF2 and ${ }^{111}$ Inor ${ }^{177}$ Lu-IMP288 showed rapid and selective tumor targeting with high tumor-to-background contrast as early as $1 \mathrm{~h}$ after injection, leading to the detection of tumors as small as $2 \mathrm{~mm}$. The activity in the tumor lesions as measured with SPECT correlated well with uptake measured with ex vivo biodistribution. In a previous study, we showed that successive administrations of PRIT within a 3-d interval resulted in similar uptake in the tumors (5). Therefore, images acquired after diagnostic or therapeutic administrations of PRIT can be compared quantitatively. Furthermore, tumor size could be derived accurately from the activity uptake (\%ID) measured with SPECT, because in small tumor lesions the uptake $(\% \mathrm{ID} / \mathrm{g})$ is relatively independent of tumor size, thus allowing the monitoring of tumor growth. The delayed 


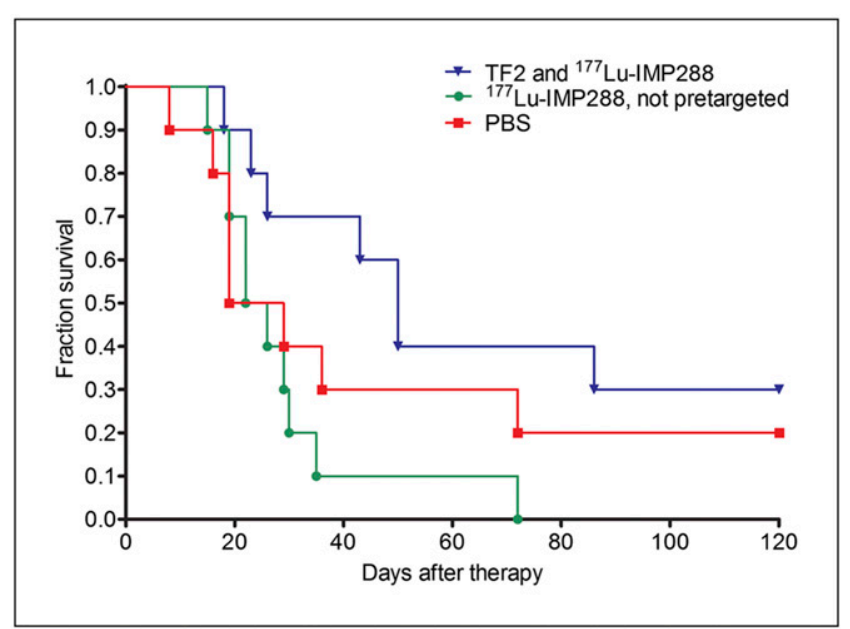

FIGURE 7. Kaplan-Meier survival curves for tumor-bearing mice treated with TF2-pretargeted ${ }^{177} \mathrm{Lu}-\mathrm{IMP} 288$, mice treated with ${ }^{177} \mathrm{Lu}-\mathrm{IMP} 288$ alone, or untreated mice (PBS). PRIT resulted in median survival of $50 \mathrm{~d}$ (95\% confidence interval, 41-59 d), whereas mice in nonpretargeted and PBS groups had median survival of 22 and $19 \mathrm{~d}$, respectively (95\% confidence interval, 15-29 and 9-29 d, respectively).

tumor growth due to PRIT corresponded to the prolonged survival in the treated group. Hence, this report shows the feasibility and additional value of imaging to monitor the efficacy of PRIT with the same pretargeting agents, TF2 and radiolabeled IMP288.

The orthotopic LS174T human colon carcinoma model for peritoneal disease has been characterized previously (17). This animal model is more relevant than the widely used models of subcutaneously growing tumors to investigate the sensitivity of an imaging method, because background activity levels can be better appreciated. Furthermore, the model is also more clinically relevant for monitoring therapy effects.

We radiolabeled IMP288 with ${ }^{111}$ In for pretargeted immunoSPECT because ${ }^{111} \mathrm{In}$ is used as a validated surrogate for ${ }^{177} \mathrm{Lu}$ (the 2 radionuclides have the same in vivo behavior). Both radionuclides are residualizing radiometals. Therefore, dosimetry can be performed with ${ }^{111}$ In data to estimate the radiation dose to the tumor. For the calculation of absorbed doses, imaging during several days is required to determine the residence time of the radiolabel in the tissues.

It would have been interesting to estimate tumor and normalorgan doses. However, for dosimetry, multiple scans $(\geq 3)$ are required within $7 \mathrm{~d}$ after injection to calculate the area under the curve for tumor and organ uptake. Multiple sessions of general anesthesia within several days would have negatively affected the condition of the mice, in turn affecting toxicity and survival data. Therefore, we decided to acquire only 1 SPECT scan at $1 \mathrm{~h}$ after injection and not to perform dosimetry in this animal study. In patients, multiple scans can easily be acquired to estimate the radiation dose to the tumor lesions and to the dose-limiting tissues.

The half-life of ${ }^{111}$ In perfectly matches the biologic halflife of the radiolabeled peptide. Former studies showed pre- targeted immuno-PET and -SPECT with radionuclides, such as ${ }^{68} \mathrm{Ga},{ }^{18} \mathrm{~F},{ }^{124} \mathrm{I}$, and ${ }^{99 \mathrm{~m}} \mathrm{Tc}(7,12-14)$, but these methods cannot be used for dosimetric calculations for therapy. The half-life of ${ }^{68} \mathrm{Ga}(68 \mathrm{~min})$ is not matched with the therapeutic radionuclide. Using ${ }^{99 \mathrm{~m}} \mathrm{Tc}$ for dosimetry on immuno-SPECT images is not feasible either, because ${ }^{99 \mathrm{~m}} \mathrm{Tc}$ clears more rapidly from the (tumor) cells after binding and internalization, in contrast to (residualizing) radiometals such as ${ }^{111} \mathrm{In}$ and ${ }^{177} \mathrm{Lu}$.

The established valuable combination of imaging and therapy, designated as theranostics, could be a useful strategy in the clinical practice of PRIT. The therapeutic efficacy of PRIT with TF2 and ${ }^{177}$ Lu-IMP288 was demonstrated previously in mice with subcutaneous LS174T xenografts (5). Furthermore, several clinical studies with bsmAb and ${ }^{111} \mathrm{In}$ labeled haptens for pretargeted immuno-SPECT showed high sensitivity (18-21). In patients, immuno-SPECT can be used to guide individual treatment, because this imaging modality can be repeated at multiple time points. In a pretherapy diagnostic cycle, tumor targeting can be demonstrated in an imaging cycle with ${ }^{111}$ In-labeled peptide. Dosimetric analysis of multiple pretherapy diagnostic scans can be used to adjust activity doses for subsequent therapy cycles. Furthermore, images can be acquired directly after therapy to observe the distribution of therapeutic injections and calculate their absorbed doses. In the follow-up period after therapy, the effect on disease progression can be evaluated by implementing a diagnostic cycle.

In our institution, a phase I clinical study of pretargeted radioimmunotherapy with TF2 and ${ }^{177} \mathrm{Lu}$-IMP288 in colorectal cancer patients is ongoing. In this study, pretherapy diagnostic cycles with TF2 and ${ }^{111}$ In-IMP288 are used for dosimetric calculations. Preliminary results of this clinical study for theranostics in PRIT are encouraging. This and other future studies will provide more information on doseresponse rates, the predictive value of dosimetry, and how dosimetry can contribute to more personalized medicine, or theranostics, in targeted radionuclide therapy.

\section{CONCLUSION}

The present study showed that pretargeted immunoSPECT with TF2 and ${ }^{111} \mathrm{In}$ - or ${ }^{177} \mathrm{Lu}$-IMP288 can be used to predict and confirm tumor uptake and monitor the therapeutic effect of pretargeted radioimmunotherapy.

\section{DISCLOSURE STATEMENT}

The costs of publication of this article were defrayed in part by the payment of page charges. Therefore, and solely to indicate this fact, this article is hereby marked "advertisement" in accordance with 18 USC section 1734.

\section{ACKNOWLEDGMENTS}

We thank Bianca Lemmers-van de Weem, Iris LamersElemans, Henk Arnts, and Jonathan Disselhorst for technical assistance. The work was supported by the Dutch Cancer Society (KWF Kankerbestrijding, grant KUN 2008-4038) and 
the National Institutes of Health (National Institute of Biomedical Imaging and Bioengineering grant R43 EB003751). William J. McBride, David M. Goldenberg, and Chien-Hsing Chang are employed by or have financial interest in Immunomedics, Inc., or IBC Pharmaceuticals, Inc. No other potential conflict of interest relevant to this article was reported.

\section{REFERENCES}

1. Baum RP, Rösch F. 1st World Congress on Ga-68 and peptide receptor radionuclide therapy (PRRNT); June 23-26, 2011; Zentralklinik Bad Berka, Germany. World J Nucl Med [serial online]. 2011;10:5-6. Available from: http:// www.wjnm.org/text.asp?2011/10/1/5/82105. Accessed October 12, 2012.

2. Goldenberg DM, Chang C-H, Rossi EA, Mcbride WJ, Sharkey RM. Pretargeted molecular imaging and radioimmunotherapy. Theranostics. 2012;2:523-540.

3. Le Doussal JM, Martin M, Gautherot E, Delaage M, Barbet J. In vitro and in vivo targeting of radiolabeled monovalent and divalent haptens with dual specificity monoclonal antibody conjugates: enhanced divalent hapten affinity for cell-bound antibody conjugate. J Nucl Med. 1989;30:1358-1366.

4. Karacay H, McBride WJ, Griffiths GL, et al. Experimental pretargeting studies of cancer with a humanized anti-CEA x murine anti-[In-DTPA] bispecific antibody construct and a ${ }^{99 \mathrm{~m}} \mathrm{Tc}-/^{188}$ Re-labeled peptide. Bioconjug Chem. 2000;11:842-854.

5. Schoffelen R, van der Graaf WT, Franssen G, et al. Pretargeted ${ }^{177}$ Lu radioimmunotherapy of carcinoembryonic antigen-expressing human colonic tumors in mice. J Nucl Med. 2010;51:1780-1787.

6. Sharkey RM, Cardillo TM, Rossi EA, et al. Signal amplification in molecular imaging by pretargeting a multivalent, bispecific antibody. Nat Med. 2005;11:1250-1255.

7. Sharkey RM, Karacay H, Vallabhajosula S, et al. Metastatic human colonic carcinoma: molecular imaging with pretargeted SPECT and PET in a mouse model. Radiology. 2008;246:497-507.

8. Sharkey RM, McBride WJ, Karacay H, et al. A universal pretargeting system for cancer detection and therapy using bispecific antibody. Cancer Res. 2003;63:354-363.

9. Griffiths GL, Chang $\mathrm{CH}$, McBride WJ, et al. Reagents and methods for PET using bispecific antibody pretargeting and ${ }^{68} \mathrm{Ga}$-radiolabeled bivalent haptenpeptide-chelate conjugates. J Nucl Med. 2004;45:30-39.
10. Karacay H, Sharkey RM, McBride WJ, Rossi EA, Chang CH, Goldenberg DM. Optimization of hapten-peptide labeling for pretargeted immunoPET of bispecific antibody using generator-produced ${ }^{68} \mathrm{Ga}$. J Nucl Med. 2011;52:555-559.

11. McBride WJ, D'Souza CA, Sharkey RM, et al. Improved ${ }^{18} \mathrm{~F}$ labeling of peptides with a fluoride-aluminum-chelate complex. Bioconjug Chem. 2010;21:1331-1340.

12. Schoffelen R, Sharkey RM, Goldenberg DM, et al. Pretargeted immuno-positron emission tomography imaging of carcinoembryonic antigen-expressing tumors with a bispecific antibody and a ${ }^{68} \mathrm{Ga}$ - and ${ }^{18} \mathrm{~F}$-labeled hapten peptide in mice with human tumor xenografts. Mol Cancer Ther. 2010;9:1019-1027.

13. Schoffelen R, van der Graaf WT, Sharkey RM, et al. Pretargeted immuno-PET of CEA-expressing intraperitoneal human colonic tumour xenografts: a new sensitive detection method. EJNMMI Res. 2012;2:5-14.

14. McBride WJ, Zanzonico P, Sharkey RM, et al. Bispecific antibody pretargeting PET (immunoPET) with an ${ }^{124}$ I-labeled hapten-peptide. J Nucl Med. 2006;47:1678-1688.

15. Rossi EA, Goldenberg DM, Cardillo TM, McBride WJ, Sharkey RM, Chang CH. Stably tethered multifunctional structures of defined composition made by the dock and lock method for use in cancer targeting. Proc Natl Acad Sci USA. 2006;103:6841-6846.

16. Goldenberg DM, Rossi EA, Sharkey RM, McBride WJ, Chang CH. Multifunctional antibodies by the Dock-and-Lock method for improved cancer imaging and therapy by pretargeting. J Nucl Med. 2008;49:158-163.

17. Koppe MJ, Soede AC, Pels W, et al. Experimental radioimmunotherapy of small peritoneal metastases of colorectal origin. Int J Cancer. 2003;106:965-972.

18. Aarts F, Boerman OC, Sharkey RM, et al. Pretargeted radioimmunoscintigraphy in patients with primary colorectal cancer using a bispecific anticarcinoembryonic antigen CEA $X$ anti-di-diethylenetriaminepentaacetic acid $F(a b ') 2$ antibody. Cancer. 2010;116:1111-1117.

19. Barbet J, Peltier P, Bardet S, et al. Radioimmunodetection of medullary thyroid carcinoma using indium-111 bivalent hapten and anti-CEA $\mathrm{x}$ anti-DTPA-indium bispecific antibody. J Nucl Med. 1998;39:1172-1178.

20. Le Doussal JM, Chetanneau A, Gruaz-Guyon A, et al. Bispecific monoclonal antibody-mediated targeting of an indium-111-labeled DTPA dimer to primary colorectal tumors: pharmacokinetics, biodistribution, scintigraphy and immune response. J Nucl Med. 1993;34:1662-1671.

21. Chetanneau A, Barbet J, Peltier P, et al. Pretargetted imaging of colorectal cancer recurrences using an ${ }^{111} \mathrm{In}$-labelled bivalent hapten and a bispecific antibody conjugate. Nucl Med Commun. 1994;15:972-980. 